\title{
OPTIMIZING LASER SCANNING POSITIONS IN BUILDINGS EXTERIORS: HERITAGE BUILDING APPLICATION
}

\author{
Mahmoud METAWIE, Mohamed MARZOUK $\mathbb{D D}^{*}$ \\ Structural Engineering Department, Faculty of Engineering, Cairo University, Giza, Egypt
}

Received 12 September 2019; accepted 10 January 2020

\begin{abstract}
Digital documentation for heritage buildings is one of the methods of preserving them as it provides a current record for the buildings. Digital records of heritage buildings can be used for future building rehabilitation, or be presented to the public to raise the awareness, increase tourism and decrease vandalism. This paper focuses on scanning object geometry factor to increase the quality of heritage's façade point cloud. It optimizes the scanner locations and the scanner field of view to increase the point cloud quality and shorten the scanning time while guaranteeing a set of quality constraints for the point cloud. The quality constraints are based on the incidence angle between the scanned surface and the laser beam, and the max spacing between points. Three different multi-objective optimization algorithms are utilized: 1) genetic algorithm, 2) Jaya algorithm, and 3) particle swarm optimization to increase the quality. Optimization performance measures are adopted to compare the outputs of the optimization algorithms. A multi-criteria decision-making technique (Weighed sum model) is used to choose the optimum solution between the Pareto frontier solutions. Optimization algorithms minimize point cloud density and scanning time while assuring a required point spacing and max incidence angle by changing distance between laser scanner and scanned Facade, horizontal and vertical scan repetitions, and scanner different resolutions. The Jaya algorithm generates the most diversifiable optimal solutions and it is the fastest of the three algorithms considered. This research focuses on vertical building façade and future research will include the all types of Heritage façade. Omar Tosson Palace in Egypt is considered as a case study to demonstrate the use of the developed methodology and to illustrate its essential features.
\end{abstract}

Keywords: laser scanning, heritage documentation, point cloud, optimization, multi-criteria decision-making.

\section{Introduction}

Cultural heritage buildings represent a country's identity and history. Egyptian heritage buildings can be categorized, according to their age, as Pharaonic, Greek and Romanian Buildings, Christian buildings, as well as Islamic buildings. Heritage buildings are susceptible to high levels of deterioration, hence should be continuously maintained and restored. Deterioration in Egyptian heritage buildings is mainly attributed to three main factors: 1) human factors such as accidents, fires, ignorance, negligence, theft, and vandalism, 2) natural factors including erosion, forces of nature, climate changes, underground water, and 3) lifespan factor since most of the heritage buildings were built long time in the past. The initial step of maintaining and restoring a heritage building is to document the current state of these buildings. Terrestrial Laser Scanning (TLS) is an advanced digital technique that improves the accuracy and the precision of capturing and recording Heritage buildings. In order to construct a full 3D point cloud data of a heritage building with a laser scanner, multiple scans from different locations and orientations are required. This is to overcome occlusions, scan range and scan view limits. The set of scanning positions, orientations, and scanning parameters must be carefully configured so that the point cloud and the 3D model can be reasonably reconstructed with a minimal number of scans and superior quality. The use of TLS for heritage documentation has become increasingly popular. Terrestrial laser scanners have been used for documenting large historic monuments or sites, such as the project on Forum of Pompeii (Balzani et al., 2004), Great Buddha projects (Ikeuchi et al., 2007), Romanian Heritage Monuments (Calin et al., 2015), and Nasif Historical House in Jeddah (Baik, 2017).

Sánchez-Aparicio et al. (2018) studied preservation of heritage sites by combining TLS data and radiometric.

${ }^{*}$ Corresponding author. E-mail: mm_marzouk@yahoo.com 
They developed a method capable of determining chemical, biological and physical damages in historical masonry constructions. They validated their study by choosing the Fortress of Almeida, in Portugal as a case study. Antón et al. (2018) assessed the accuracy of 3D modeling for historical building information models created by point cloud data and BIM tools. They identified a three stage semi-automatic process to generate Heritage building information modelling (HBIM) using point cloud and BIM tools. Shanoer and Abed (2018) studied the registration of point clouds for documenting cultural heritage buildings. Two registration methods were compared in order to evaluate TLS outputs for documenting cultural heritage. The accuracy of documentation was assessed based on combining TLS outputs and processing elapse time factor. Shao et al. (2019) automated registration of point clouds resulting from structured light scanner and TLS for documentation of heritage. TLS was bundled with photogrammetry for better visualization as reported in literature (Alshawabkeh \& Haala, 2004; Cabrelles et al., 2009; El-Hakim et al., 2004; Grussenmeyer et al., 2008; Guidi et al., 2009; Remondino, 2011; Yastikli, 2007). Moreover, TLS has been bundled up with Virtual Reality to better visualize heritage (Fernández-Palacios et al., 2017; Tan et al., 2016; Basantes et al., 2017; Tschirschwitz et al., 2019; Forte et al., 2004; Kersten et al., 2017, 2018a, 2018b).

This paper sheds light on scan geometry factors, aiming at enhancing the point cloud quality resulting from scanning heritage buildings. TLS is used as a heritage recording tool to capture the geometric characteristics of a heritage building as precisely as possible. A methodology is introduced here for optimizing the Terrestrial Laser scanner positions as well as the scanner field of view in order to improve the point cloud quality while reducing scanning, and fulfill quality constraints with respect to point cloud such as allowable incidence angle, minimum/ maximum scan range, and the field of view. These quality constraints depend on the incidence angle between the scanned surface normal and the laser beam, as well as the maximum spacing between points. The paper proposes a new technique for planning TLS for heritage facades that specifies the optimal locations of the scanner. It calculates the minimum number of scanner positions and identify their relative locations in order to minimize scanning time and point density while ensuring the satisfaction of a group of quality constraints for the point cloud. The quality restrictions are: maximum incidence angle between the façade surface normal and the laser beam, and max point spacing. Three separate multi-objective optimization algorithms were utilized: 1) genetic algorithm, 2) Jaya algorithm, and 3) particle swarm optimization to increase the quality. Optimization performance measures are used to compare the outputs of the optimization algorithms. A weighed sum model is used as the decision-making technique to choose the optimum solution between the Pareto frontier solutions. The Tosson Palace in Egypt is used as a case study to demonstrate the use of the developed methodology and to illustrate its essential features.

\section{Literature review}

A key factor in scanning heritage is knowing the point density and measurement accuracy necessary to generate the level of deliverable data for the project at hand (Barber et al., 2003). Scanner specifications as well as the scanning configuration and conditions are key attributes of measurement accuracy. Soudarissanane et al. (2009) classified the factors affecting data accuracy as follows: instrument mechanism, atmospheric conditions, object surface properties, and scanning geometry. Scanning object geometry depends on the position and orientation of the required scanned surface with reference to the TLS position. The position of the TLS specifies the range, incidence angle, and the point density of the produced laser points. The main aim is to develop a high-quality point cloud with a minimum scanning time. The point cloud quality depends mainly on the positions of the scanner relative to the scanned surface. There are several factors affecting that, some of them are beyond the operator control such as the characteristics of the scanner, the environmental conditions during the scanning or the surface materials (Soudarissanane et al., 2009; Lee et al., 2010). On the other hand, there are other factors affecting the point cloud quality that are within the control of the operator, such as the position of the scanner relative to the scanned surface or the point density (Soudarissanane et al., 2009). Soudarissanane and Lindenbergh (2011) performed a study to determine optimum scan locations of a TLS in a simulated scene. They defined the minimum number of views that achieve full coverage with good data quality and smaller propagation errors. Kersten et al. (2009) investigated the accuracy behavior of the 3 rd generations of terrestrial laser scanning systems. They utilized different tests for geometric accuracy of different terrestrial laser scanning systems such as: 3D accuracy field test, distance measurements test, inclination compensation test, and incidence angle influence on laser beam test. Tang and Alaswad (2012) investigated the issue of finding optimal scan positions in as-built documentation of buildings. They suggested an optimization method that guarantees that all information is gathered with the required level of detail. For this method to work, the authors established a model that creates relationships between some parameters related to data quality (e.g. accuracy and point cloud density), and others related to the method of data collection (e.g. height or distance of the scanner). Similarly, Song et al. (2014) proposed a laser scanning methodology of scanning building façades aimed at minimizing the number of scans while achieving a specific level of detail. They proposed a model to produce spherical spaces that are feasible to allow capturing features with the required detail level. These spaces are characterized by internal parameters such as scanner resolution, as well as external parameters such as scanning positions or the shapes of the features. To be able to reduce the time needed to cover all potential spaces for all features, they grouped related features into clusters. The results are then represented on a heat map that illustrates 
the number of features captured from candidate scanning positions for a given level of detail. Zhang et al. (2016) conducted TLS in dynamic construction site environments to achieve the required data quality. They proposed a "divide-and-conquer" strategy by producing graphs with nodes constituting the quality requirements (e.g., accuracy and level of detail) linked by edges that highlight the spatial relationships between the nodes. The conclusion was that their approach improved the work done by experienced professionals in TLS. In a related context, in their research efforts Kriegel et al. (2015) and Blaer and Allen (2009) designed iterative methods to calculate the nextbest-scans, taking into consideration the model quality generated from a TLS located on a self-directed robot. Giussani and Scaioni (2004) developed techniques for planning TLS on specific environments such as cultural heritage. Ahn and Wohn (2015) proposed a way to find proper scanner configurations taking into consideration many constraints such as angle of incidence, full coverage, sufficient overlap, and scan range limit. Similarly, point density of the scanned surface is often considered a measurement quality indicator. A minimum density (minimum distance between consecutive points at a certain distance from the sensor) is usually selected beforehand. Cabo et al. (2017) devised a new technique for planning TLS measurements that determines the best locations of the scanner for scanning heritage facades.

Previous research investigated the quality of point cloud based on incidence angle, and distance on 2D discretized map of the scene and they did not study the effect of the height of the building. Neither did they use optimizations algorithms such as genetics algorithm, or particle swarm to provide optimal viewpoints. This research supplied a new method to determine the best positions of the scanner to scan a heritage façade with high quality. It achieved high point cloud data quality based on incidence angle, and max spacing between points constraints, while achieving minimum point density, and scanning time by using different optimization techniques.

\section{Optimization algorithms}

\subsection{Optimization of genetic algorithms}

Genetic algorithms (GA) are evolutionary algorithms, established in 1975 by John Holland (Matthias et al., 2013). It is comprised of two key processes. First one is the process of selecting individuals to produce next generation. The second process is manipulating the chosen individuals to procedure the following generation by crossover and mutation. The selection approach determines the choice of the chromosomes used in reproduction and the number of off springs that are produced. The superior individual has a better chance of being a parent. The initial stage is to create random solutions called chromosomes. At each stage, a new population is formed and is called "generation". Chromosomes are made of genes that carry a group of values to the optimization variables. The second stage is calculating the "fitness function" for each population's chromosome. The fitness function helps in the assessment of distinct chromosomes. The third stage is the chromosomes selection. There are different strategies of chromosomes selection: the roulette wheel selection, the rank selection, the steady-state selection, the elitism, the Boltzmann selection, and the tournament selection strategy. The aforementioned selection controls the creation of the new chromosomes. The fourth stage is performing the crossover to create a new child from the two chromosomes or individuals. The different types of crossover include single-point, two-point, or uniform crossover. Single-point crossover is the most popular type. A random single point is chosen from one parent to be exchanged to the other parent. The fifth stage is performing the mutation process. A mutation gene is randomly selected and mutated by a small amount, or replaced by a new amount. The purpose of mutation is to ensure genetic diversity within the population, and to avoid the stagnation around local minimal. Finally, a population is produced for each generation and previous procedures carry on for a specified number of iterations. Optimum solution is the chromosomes in the final iteration.

Non-dominated Sorting Genetic Algorithm II (NSGAII) is used in this paper to determine the optimal solution. It is a multi-objective genetic algorithm, proposed by Deb et al. (2002). In addition to the previous illustrated genetic operators two specialized operators in NSGA-II is utilized:

- Non-dominated sorting: the population is organized and divided into fronts $\left(F_{1}, F_{2}\right.$, etc.), where $F_{1}$ (first front) represents the approximated Pareto front. Sorting the population into several ranks (fronts) is based on the concept of dominance as per the following: a solution (a) is considered dominant over the other solution (b) provided that solution (a) is not worse than solution (b) in respect to all the objectives, and solution (a) is considerably better than solution (b) in one objective at least. In the case that any of the two conditions are not met, then solution (a) does not dominate solution (b).

- Crowding distance: it is a factor that given to every solution in the population in order to measure the density of solutions around a particular solution $i$. Therefore, the average distance between two solutions adjacent to solution $i$ are estimated for all $M$ objectives.

These ranking mechanisms are used, to create the population of next generation. A MATLAB code was written using MATLAB (2018) in order to develop the multiobjective Non-dominated Sorting Genetic Algorithm II optimization.

\subsection{Quasi-oppositional Jaya algorithm}

Multi-objective quasi-oppositional Jaya MOQO-Jaya algorithm is developed to solve the multi-objective optimization problems (Rao et al., 2017). The MOQO-Jaya algorithm is an updated version of MO-Jaya algorithm. 
The concept of opposition-based learning is used, to expand the population and enhance the convergence rate of MOQO-Jaya algorithm. In order to accomplish better approximation, another population opposed to the current one is created according to Eqn (1):

$$
n_{\text {new }}=\operatorname{round}\left(n_{\text {old }}+r^{*} n_{\text {old }}\right) \text {, }
$$

where $r$ is a random value in the interval $[-0.5,0.5]$. The population size can increase or decrease as a result of the positive or negative random value of $r$. In MOQO-Jaya algorithm the solutions are updated similar to the developed Jaya algorithm based on Eqn (2):

$$
\begin{aligned}
& A(i+1, j, k)=A(i, j, k)+r(i, j, 1)(A(i, j, b)- \\
& |A(i, j, k)|)-r(i, j, 2)(A(i, j, w)-|A(i, j, k)|),
\end{aligned}
$$

where $b$ and $w$ represent best and worst solutions index for the current created population. $r(i, j, 1)$ and $r(i, j, 2)$ are random numbers between $[0,1]$. These random numbers are used for scaling and guaranteeing good diversification. $i, j, k$ represent the number of iterations, number of variables and number of candidate solutions, respectively. $A(I$, $j, k$ ) represents the $j$ variable of $k$ candidate solution in the iteration $i$. Nonetheless, in order to solve multiple objectives problems efficiently the MOQO-Jaya algorithm is associated with non-dominated sorting methodology as well as crowding distance computation mechanism. To solve single-objective optimization, it is quite simple to determine which solution is better from the objective function

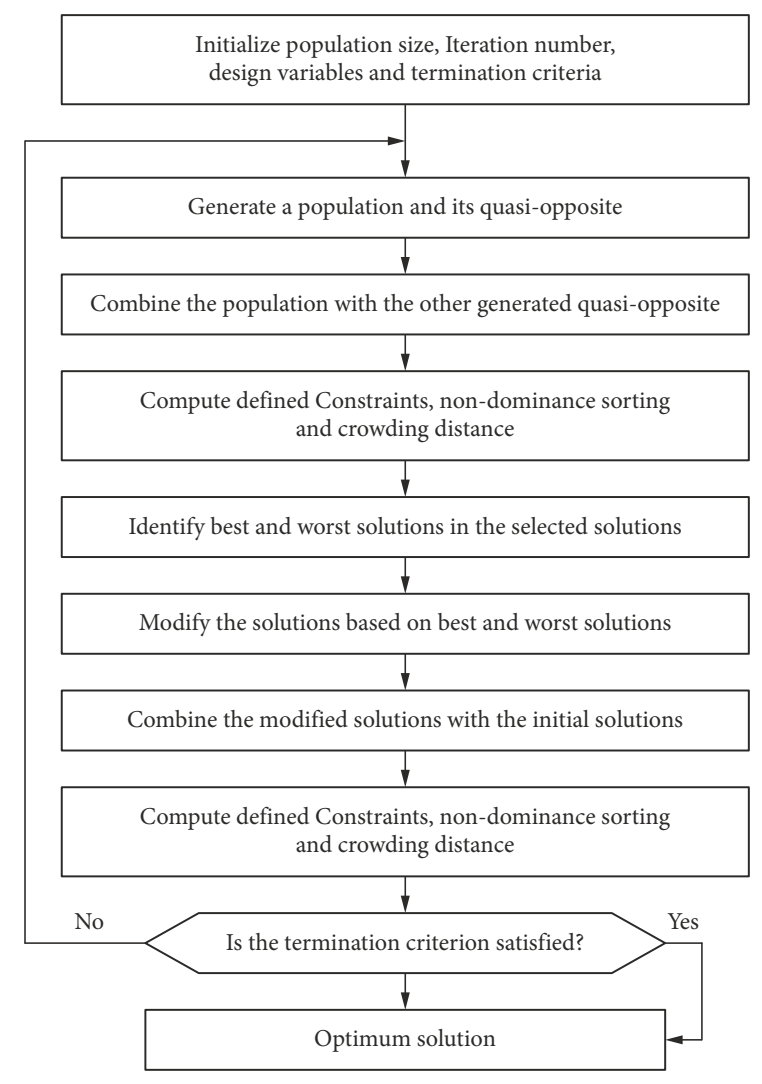

Figure 1. Flowchart of MOQO-Jaya algorithm value. However, in the case of multiple conflicting objectives, it's difficult to distinguish between the worst and best solutions from a group of solutions (see Figure 1).

In the MOQO-Jaya algorithm, selecting the worst and best solutions is done by comparing the ranks given to all solutions according to the crowding distance value, constraints-dominance concept, and non-dominance concept. As a start, an initial random population is formed. Afterwards, a quasi-opposite population is produced which is combined with the initial population. The resulted new population is sorted and ranked by applying the nondominance concept and computing the crowding distance for every solution. Following this, the $P$ best solutions are chosen from the combined population according to the rank and crowding distance. Furthermore, the solutions are updated according to Eqn (14) of the Jaya algorithm. To do this, the worst and best solutions need to be identified. The highest ranked solution ( rank =1) is chosen as the best solution. Conversely, the lowest rank solution is chosen as the worst one. In the case of having two solutions or more with the same rank, the solution of the highest crowding distance value is selected as the best solution. After updating all solutions, the new solutions are combined with the initial population. Afterwards, these solutions are sorted and ranked again by applying the non-dominance concept and computing the crowding distance value for each solution. A number $P$ of best solutions are then selected according to the new rankings and crowding distance values. The superior solutions are selected based on the corresponding non-dominance ranks and the crowding distance values.

\subsection{Particle swarm optimization}

Particle swarm optimization (PSO) is a heuristic population-based search algorithm that was first established in 1995 by Eberhart and Kennedy. PSO simulates the bird's social behavior when flocking to the preferred place. PSO starts by forming a population called "swarm" which is comprised of individuals or "particles" where each particle flies according to its own as well as its companions' flying experience. Each particle is a possible solution in a multi-dimensional space. The status of each particle is determined by its velocity and position and they're both updated with every repetition (Zhang \& Li, 2010). PSO produce a population of solutions called "particles". Updating particles is done through exchanging information between them. Each particle flies in the multi-dimensional space towards its personal best "pbest" flying experience, as well as the best experience available in the whole swarm (global best) "gbest". During optimization, the position and the velocity are updated for each particle in the population using Eqns (3) and (4), respectively:

$$
\begin{aligned}
& v_{i}(\mathrm{t}+1)=w \times v_{i}(t)+c_{1} \times r_{1} \times\left(\text { pbest }_{i}(t)-\right. \\
& \left.x_{i}(t)\right)+C_{2} \times r_{2} \times\left(\text { gbest }_{i}(t)-x_{i}(t)\right) ; \\
& x_{i}(t+1)=x_{i}(t)+v_{i}(\mathrm{t}+1) .
\end{aligned}
$$


In these equations $x_{i}(t+1)$ is the updated particle $i$ position. $x_{i}(t)$ represents the particle $i$ current position. $v_{i}(t+1)$ represents the updated particle $i$ velocity. $v_{i}(t)$ represents the particle i current velocity. $r_{1}$ and $r_{2}$ are uniformly random distributed numbers between $[0,1]$ that represent a potential way to find better solutions towards the direction of the personal best, and the global best. $c_{1}$ and $c_{2}$ are constants that are normally assumed and deal with social parameters, and the cognitive learning that govern the effects of global and personal guides. $w$ is the inertia weight which controls the balance between the personal, and the global experiences. It ranges between 0.3 , and 0.7 and usually recommended to initially have a large inertia weight, then it is reduced during the optimization process by a damping factor to easily explore the entire search space. A MATLAB code was written in order to carry out this optimization procedure.

\section{Model development}

\subsection{Problem formulation}

The multi-objective optimization model is used to determine the optimal solutions using three different optimization techniques which are: (1) genetic algorithm, (2) JAYA algorithm, and (3) particle swarm optimization. The objective of optimization is to minimize point cloud density and scanning time while assuring a required point spacing and maximum incidence angle by changing distance between laser scanner and scanned surface, horizontal and vertical scan repetitions to full facade coverage, and Scanner different resolutions.

\subsubsection{Decision variable}

TLS provides a $3 \mathrm{D}$ point cloud of a scene by transmitting laser beams to an object or surface and receiving it back. It specifies the distance $(\rho)$ between the laser scanner and the scanned object by measuring the time required for the laser beam to fly to the object and return back to the scanner. The location of the object is determined by recording the distance $(\rho)$, and the horizontal $(\theta)$ and the vertical $(\varphi)$ angles required for the laser beam to reach the object (see Figure 2).

The main optimization decision variables are the distance between laser scanner and scanned surface, scanner different resolutions, and horizontal and vertical scan repetitions to full facade coverage. For horizontal and vertical scan repetitions, the scanner is positioned horizontally and vertically at equally spaced locations to full scan the target façade and satisfy certain requirements. Figure 3 shows an example of a TLS scanner that repeated 4 times horizontally and 3 times vertically. Scaffoldings, or scissors lifts can be used to carry the Scanner in the vertical repetitions. In this example, the scanner will be used at three different levels at each horizontal station, 12 times in total, to eliminate the shadows and satisfy certain requirements.

Accordingly, for each scanned position, there is a maximum field of view that depends on the number of the proposed repetitions as shown in Figures 3 and 4. This

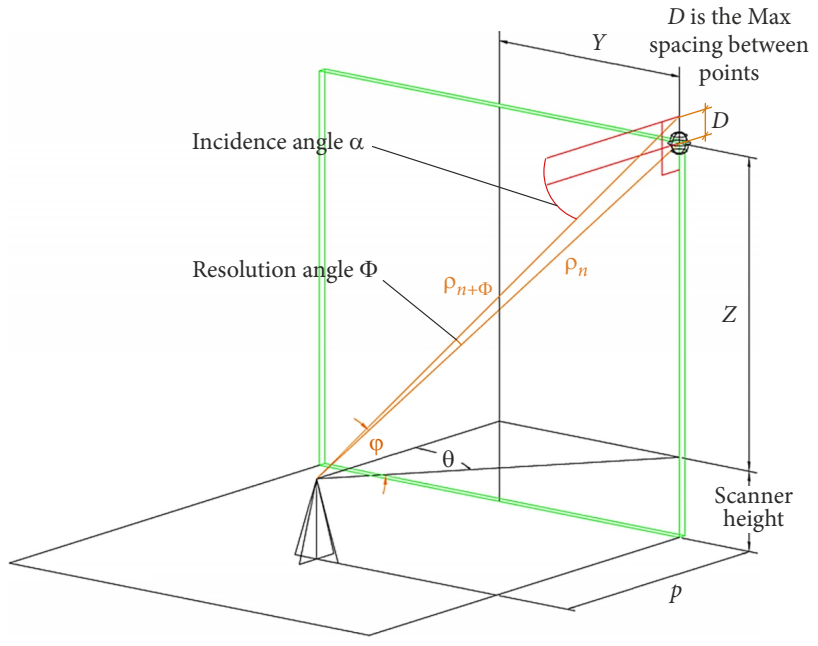

Figure 2. Scan position parameters
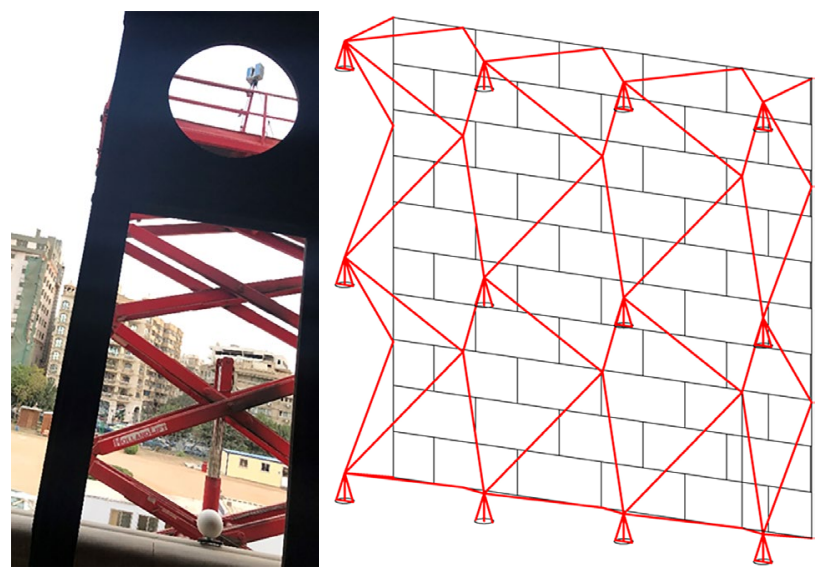

Figure 3. Scanning repetitions of the façade using scissors lift

field of view depends on the max horizontal and vertical angles for each scan position. The dimensions of field of view $(Y \& Z)$ depend on the total façade length and height, and the required horizontal and vertical repetitions as per Eqns (5) and (6) (see Figure 4).

$$
\begin{aligned}
& Y=\text { Total façade Length / } \\
& \left(2^{*}((\text { Horizontal repetition }-2)+1)\right) ; \\
& Z=(\text { Total façade height }- \text { Scanner tripod height }) / \\
& \left(2^{*}((\text { Vertical repetition }-2)+1)\right) .
\end{aligned}
$$

Also, horizontal angles $(\theta)$ and vertical angles $(\varphi)$ can be calculated as per following Eqns (7) and (8), respectively.

$$
\begin{aligned}
& \theta=\operatorname{Tan}^{-1}\left(\frac{Y}{d}\right) ; \\
& \varphi=\operatorname{Tan}^{-1}\left(\frac{Z^{\star} \operatorname{Cos} \theta}{d}\right) .
\end{aligned}
$$

$Y$ and $Z$ represent the max area covered by a scan position and $d$ is the clear spacing between the scanner and the vertical façade as shown in Figure 4. The last optimization parameter is the scanning required resolution. 


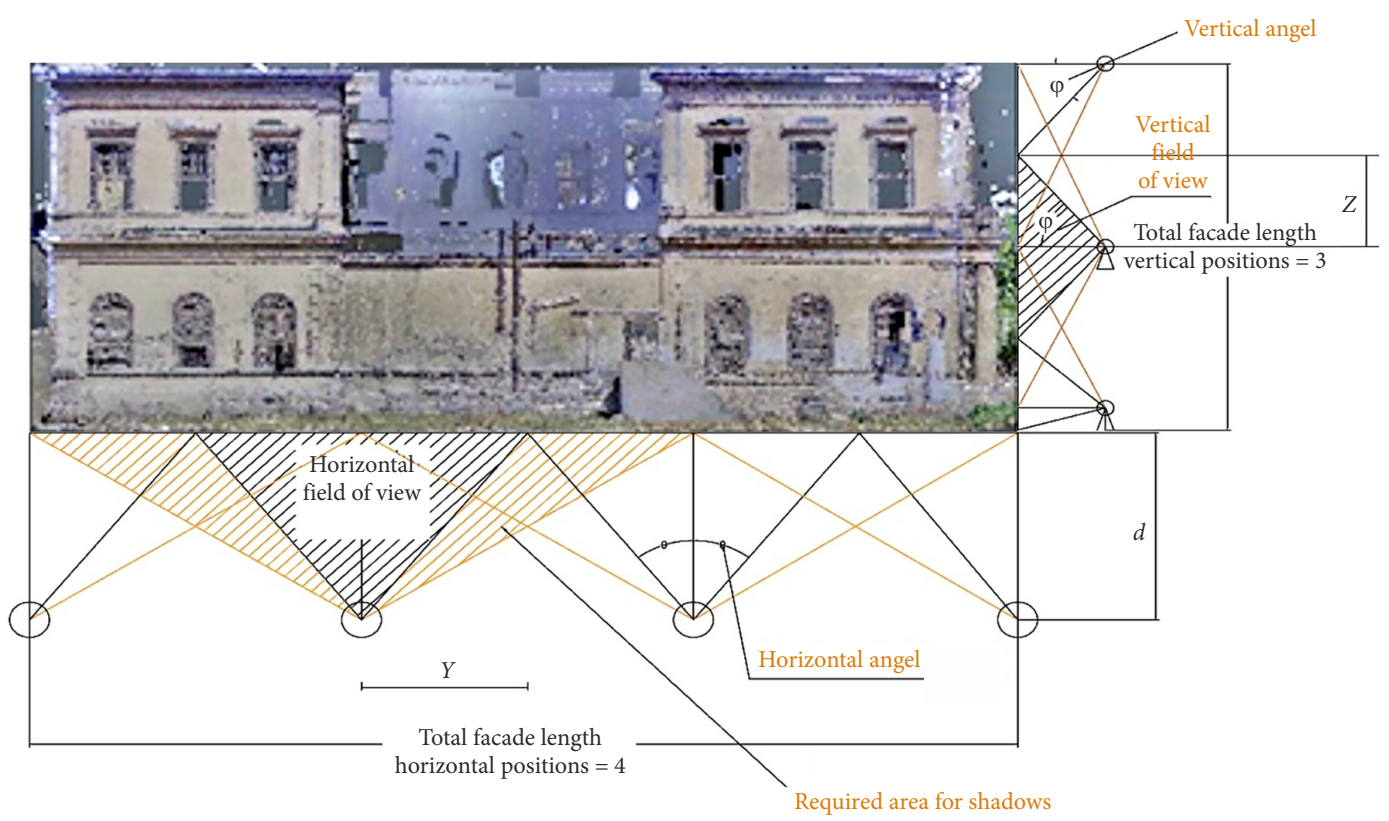

Figure 4. Scanning positions to fully cover the façade

Scanning resolutions depend mainly on the increments angle between laser signals which depends on scanner model and type (see Figure 2).

\subsubsection{Constraints}

There are two constraints for the optimization model which are incidence angle and max spacing between point in the target scanned area. Incidence angle is the angle between the laser beam and the normal of the considered surface point as depicted in Figure 2. In the proposed optimization model, incidence angle is calculated using Eqn (9).

$$
\begin{aligned}
& \alpha=\operatorname{Cos}^{-1}(d / \rho) ; \\
& \rho=d /\left(\operatorname{Cos}(\theta){ }^{\star} \operatorname{Cos}(\varphi)\right),
\end{aligned}
$$

where $\alpha$ is the incidence angle, $\rho$ is the distance between the scanner sensor and the corner of scan field area and $d$ is the clear spacing between the scanner and the vertical façade. The max incidence angle for each scan position at the target scan area border should not be greater than a specified threshold to insure the scan point precision. Soudarissanane et al. (2009) indicated that incidence angle should be less than $60^{\circ}$. The second constraint is the max spacing between points in the target scan area. This spacing is increased gradually to be maximum at the corner of the targeted scanned area. Max spacing between points for heritage facades case is considered to be varied between 4 to $6 \mathrm{~mm}$ in this research. As spacing less than $4 \mathrm{~mm}$ makes the point cloud very heavy and hard to processing and if spacing is taken more than $6 \mathrm{~mm}$ important details might be lost. The maximum point spacing $(D)$ between points depend on the maximum vertical and horizontal angles at the corner of the targeted scanned area, and on the resolution angular angle $\Phi$ and it can be calculated using Eqn (11).
$D=\rho_{\max }\left(\operatorname{Sin}\left(\varphi_{\max }\right)\right)-\rho_{\max +\Phi}\left(\operatorname{Sin}\left(\varphi_{\max }+\Phi_{\text {res }}\right)\right) ;$

$\rho_{\max }=d /\left(\left(\operatorname{Cos}\left(\theta_{\max }\right) * \operatorname{Cos}\left(\varphi_{\max }\right)\right)\right.$;

$\rho_{\max +\Phi}=d /\left(\left(\operatorname{Cos}\left(\theta_{\max }+\Phi_{r e s}\right) * \operatorname{Cos}\left(\varphi_{\max }+\Phi_{r e s}\right)\right)\right.$,

where $\rho_{\max }$ and $\rho_{\max +\Phi}$ are the lengths of the last two laser beams at the scanned area edge, $\theta_{\max }$ and $\varphi_{\max }$ are the maximum horizontal and vertical angles at the edge, and $d$ is the clear spacing between the scanner and the façade (see Figure 2).

\subsubsection{Objectives}

There are two objectives for this framework which are: 1) minimizing the scanning time and 2) minimizing point cloud density. Point cloud density is the numbers of points per meter squared. Minimizing point cloud density decreases the point cloud file size and improves the point cloud workability and decreases the processing time. Density of point cloud is very hard to calculate as the spacing between points changes in the horizontal and vertical direction continuously. To calculate density for a particular scanned area $\left(Z^{\star} Y\right)$ (see Figure 5), the number of points projected on this area is needed to be counted. For every horizontal angle step there is a unique maximum vertical angle and a unique number of vertical steps (see Figure 5). The total number of points is the summation of points for all horizontal steps as per following algorithm:

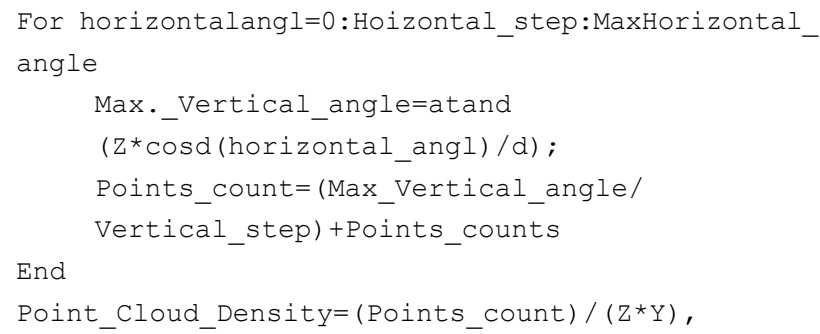




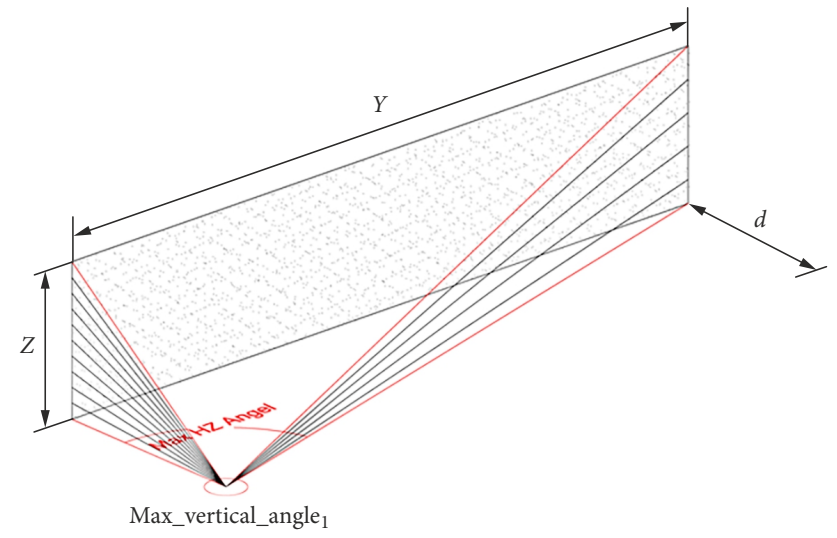

Figure 5. An example of vertical steps for two different horizontal angles for density calculations

where $Y$ and $Z$ are the scanned area dimension, $d$ is the clear spacing between the scanner and the scanned area. Point cloud density is the number of points in the point cloud per the scanned area $\left(Z^{\star} Y\right)$. Scanning time for the entire façade can be calculated using Eqn (14):

$$
\begin{aligned}
& \text { Scanning time }=\text { Vertical repetition }(\mathrm{Vr}){ }^{*} \text { Horizontal } \\
& \text { repetition }(\mathrm{Hr}){ }^{*} \text { Time per scan resolution }+ \\
& \text { Installation horizontal time }{ }^{*} \mathrm{Hr}+\text { Installation vertical } \\
& \text { time }{ }^{\star}\left(\left(\mathrm{Vr} r^{\star} \mathrm{Hr}\right)-\mathrm{Hr}\right) .
\end{aligned}
$$

\subsection{Optimization performance measures}

In order to determine any multi-objective optimization algorithm performance there are many performance measures could be used. Two performance measures were used and they are as follows.

\subsubsection{Coverage}

This performance indicator is used to compares between two groups of non-dominated solutions (A, B). The result is the percentage of individuals of one group that are dominated by individuals of the other set. The coverage algorithm was developed using MATLAB (2018) as depicted in Figure 6.

\subsubsection{Hypervolume}

The hypervolume $(H V)$ is a measure used to assess the Pareto-fronts quality resulting from solving multi-objective optimization problem. $H V$ represents the search space volume covered by a Pareto-front resulting from a particular algorithm relating to a specific point. Accordingly, for a particular algorithm, a higher $H V$ value is preferable as it is an indication of a high Pareto-front quality. For a Pareto-front comprising $Q$ solution, a hypervolume $v_{i}$ is measured for each solution $i$ belonging to $Q$, while using a reference point $W$ and solutions $i$ that represents the corners of the hypercube. The associated hypervolume is calculated according to Eqn (15).

$$
H V=\operatorname{volume}\left(\bigcup_{i=1}^{|Q|} v_{i}\right) .
$$

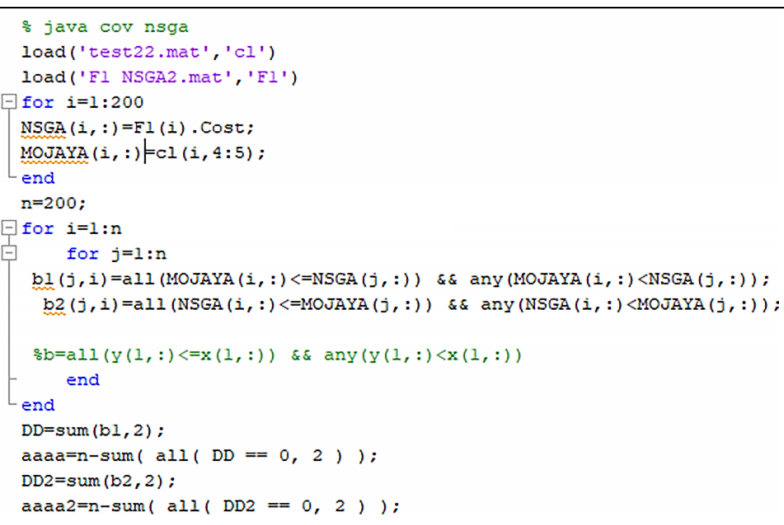

Figure 6. Coverage algorithm

In case of two objectives are considered then the calculated volume is an area. There are specific special algorithms for calculating the hypervolume such as algorithm in Beume et al. (2009). Hypervoume tool was used in MATLAB (2018) to estimate the Hypervolume indicator.

\subsection{Multi criteria decision-making}

Weighed sum model is a decision-making technique based on computing a priority index for every alternative. In a sense that the superior alternative is one with the maximum priority in the case of a maximization problem. Conversely, the optimum alternative has the minimum priority in the case of a minimization. The priority of every alternative is calculated using Eqn (16) adopted from Windarto and Muhammad (2017).

$$
p_{i}=\sum_{i=1}^{n} f_{i j} * w_{j}(1 \leq i \leq m, 1 \leq j \leq n),
$$

where $p_{i}$ is the priority of each alternative. $f_{i j}$ indicates the performance measure in the normalized matrix. $w_{j}$ is the weight of every criteria. $n$ and $m$ are the number of criteria and alternatives, respectively.

\section{Case study}

The proposed model was implemented on Tosson Palace in Egypt as shown in Figure 7 with 3500 sqm area. The main façade of the palace is $80 \mathrm{~m}$ length and $20 \mathrm{~m}$ height. $\mathrm{Z}+\mathrm{F}$ IMAGER ${ }^{\circledR} 5010 \mathrm{C}$ was used for TLS scanning and all optimization algorithms were calculated on a $2.5 \mathrm{GHz}$ Intel laptop. The multi-objective optimization module was performed based on the two objective functions previously defined in the Model Development section. In order to provide an acceptable comparison between the optimization algorithms, 10 independent optimization runs were carried out for each of the multi-objective genetic algorithm, the multi-objective Jaya algorithm, and the multi-objective particle swarm optimization. The number of iterations and the population size were assumed to equal 200, and 250 respectively for all algorithms to be able to achieve an acceptable comparison. For the genetic 


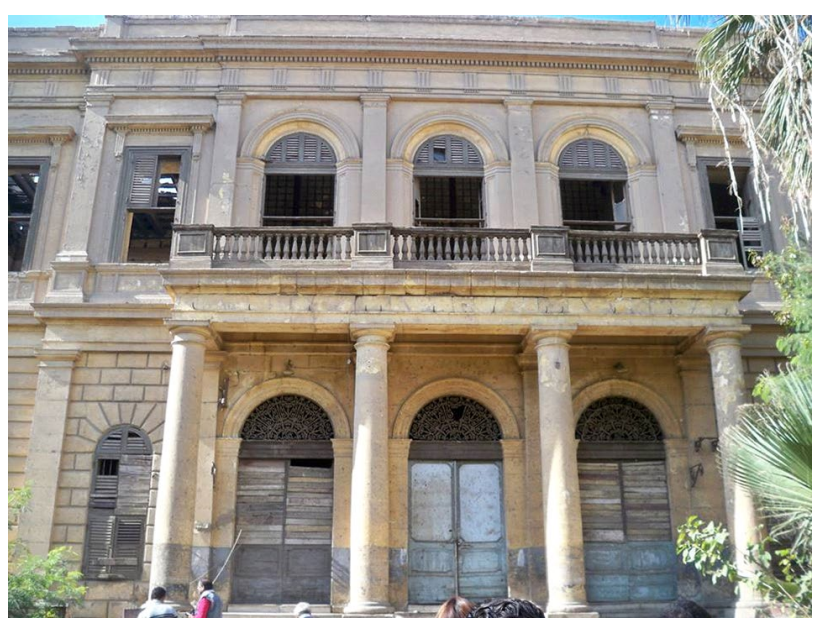

Figure 7. The front façade of Tosson Palace in Cairo- Egypt

algorithm, the tournament selection was selected as the selection strategy. Crossover rate was assumed to be 0.7 . Mutation rate was assumed to equal 0.05 . For the particle swarm optimization, the social parameters, and cognitive learning were assumed to equal 2 . The inertia weight was assumed to be 0.5 . The Jaya algorithm didn't require additional parameters aside from the population size and the number of iterations.

The range raw was considered to range from $5-20 \mathrm{~m}$. Number of horizontal and vertical repetitions that determine the scanner field of view for a specific scanner position were considered to range from 2 to 20 repetitions. The max spacing constraint was considered to range between 4 and $6 \mathrm{~mm}$ and the max incidence angle used was $60^{\circ}$. The final optimization parameter to consider was the scanning resolution. The different angular resolutions and their associated scanning time for the $\mathrm{Z}+\mathrm{F}$ laser scanner are represented in Table 1. Figure 8 shows the resulted point cloud after applying the proposed technique. It shows the front and side façades of the Palace.

Figure 9 shows a sample of Pareto frontier solutions for each optimization. Thirty-eight Pareto frontier solutions were obtained as follows; 13 solutions from the genetic algorithm, 15 solutions from JAYA algorithm, and 10 solutions from the particle swarm optimization. The blue circles, gray stars, and orange represent the Pareto frontier solutions of the genetic algorithm, Jaya algorithm,
Table 1. Scanner increment angles

\begin{tabular}{|c|c|c|}
\hline Resolution & Increments $\left(\mathrm{HZ}^{\circ} / \mathrm{V}^{\circ}\right)$ & Scanning time (mins) \\
\hline 1 & 0.2880 & 0.26 \\
\hline 2 & 0.1440 & 0.52 \\
\hline 3 & 0.0720 & 1.44 \\
\hline 4 & 0.0360 & 3.22 \\
\hline 5 & 0.0180 & 6.44 \\
\hline 6 & 0.0090 & 13.28 \\
\hline 7 & 0.0045 & 72.60 \\
\hline
\end{tabular}

Table 2. Optimization performance measures

\begin{tabular}{|l|c|c|}
\cline { 2 - 3 } \multicolumn{1}{c|}{} & Hypervolume & Jaya coverage \\
\hline JAYA & $80.00 \%$ & - \\
\hline NSGA-ll & $52.56 \%$ & $23.00 \%$ \\
\hline PSO & $75.90 \%$ & $20.00 \%$ \\
\hline
\end{tabular}

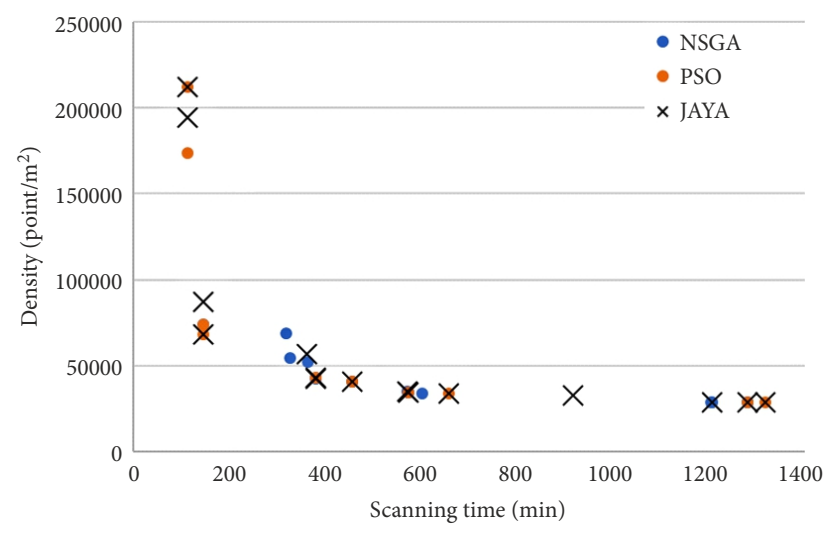

Figure 9. Sample of the Pareto frontier solutions for the three algorithms

and particle swarm optimization respectively. As shown in Figure 9, Jaya generated the most diversifiable optimal solutions. The average processing times of the JAYA algorithm, particle swarm optimization and genetic algorithm were $180 \mathrm{sec}, 260 \mathrm{sec}$, and $450 \mathrm{sec}$. Thus, JAYA had the shortest average computational time, while genetic algorithm had the longest average computational time. Table 2 shows hypervolume and coverage indicators for the optimization algorithms.

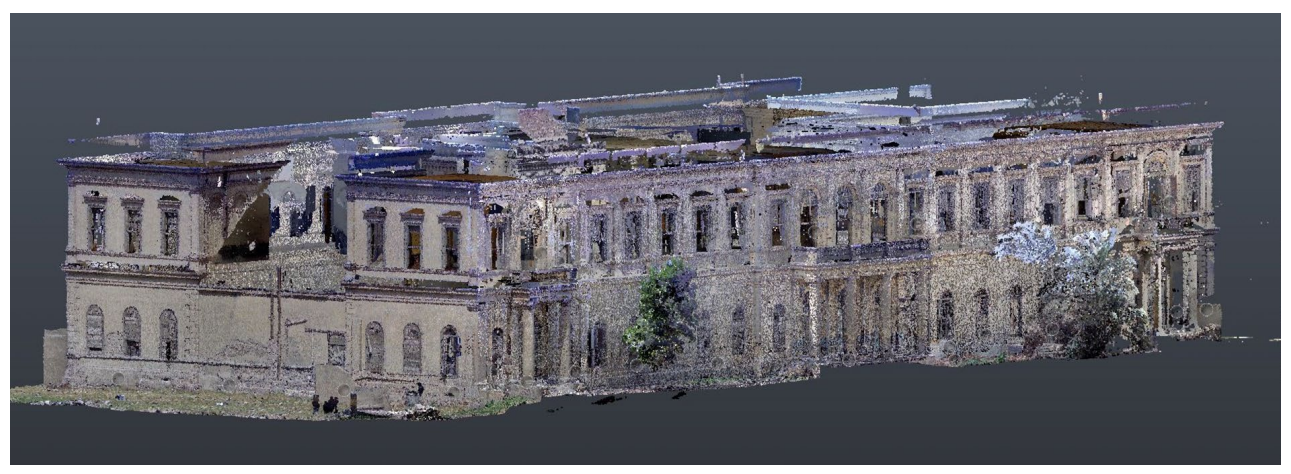

Figure 8. Tosson Palace point cloud 
Table 3. The WSM solutions' ranking

\begin{tabular}{|c|c|c|c|c|c|c|c|c|}
\hline \multicolumn{4}{|c|}{ Decision variables } & \multicolumn{2}{|c|}{ Objectives } & \multirow{2}{*}{ Algorithm } & \multirow{2}{*}{ WSM } & \multirow{2}{*}{ Ranking } \\
\hline Dis. & Hz. rep. & Vr. Rep. & Scanning resolution & Scanning time $(\min )$ & Point's density & & & \\
\hline 10 & 3 & 4 & 5 & 146.28 & 68648 & \multirow{3}{*}{ JAYA } & $80.41 \%$ & 1 \\
\hline 9 & 4 & 3 & 5 & 145.28 & 87315 & & $76.94 \%$ & 5 \\
\hline 7 & 6 & 7 & 4 & 381.24 & 42484 & & $74.65 \%$ & 7 \\
\hline 10 & 4 & 3 & 5 & 145.28 & 74236 & \multirow{3}{*}{ NSG } & $79.41 \%$ & 3 \\
\hline 6 & 6 & 6 & 4 & 325.92 & 54556 & & $74.89 \%$ & 6 \\
\hline 5 & 5 & 7 & 4 & 317.70 & 68677 & & $72.61 \%$ & 14 \\
\hline 7 & 7 & 6 & 4 & 380.24 & 43350 & \multirow{3}{*}{ PSO } & $74.53 \%$ & 11 \\
\hline 7 & 5 & 10 & 4 & 456.00 & 40943 & & $71.54 \%$ & 17 \\
\hline 8 & 7 & 9 & 4 & 573.86 & 34718 & & $67.35 \%$ & 20 \\
\hline
\end{tabular}

The decision-making module was implemented to choose the optimum solution from the generated Pareto frontier solutions. Weighed sum model was used as the decision-making technique. All Pareto frontier solutions, generated from the three algorithms, were considered as alternatives. Then, the scanning time and the density attributes were normalized according to their maximum values. Then, the associated weights were considered $60 \%$, and $40 \%$ for the scanning time, and the point density attributes respectively. A sample of the WSM solutions ranking is depicted in Table 3. The optimum solution in WSM had scanning time of 146.28 minutes, a points' density of 68648 point $/ \mathrm{m}^{2}$, with $\max$ spacing $5.77 \mathrm{~mm}$, and a $\max$ incidence angle of $44.23^{\circ}$.

\section{Conclusions}

Heritage documentation plays a very important role in heritage maintenance and condition assessment. 3D laser scanning is one of the active techniques that are utilized to document heritage buildings. However, the absence of quality definition is one of the challenges of utilizing laser scanning technique. In this paper, an optimization and decision-making model is implemented to minimize scanning time and density while achieving certain quality constraints. The terrestrial laser scanner positions are determined based on the distance between the laser scanner and the scanned surface, the required horizontal and vertical angles, and the required scanner resolution. These constraints determine the allowable point spacing and incidence angle, while minimizing the scanning time and the point density. The objective of this research is to provide a methodology to determine the optimal positions of the scanner to scan a heritage facade, based on the above- mentioned measurement limitations and constraints. Accordingly, it has to ensure that the full coverage of the facade surface with the minimum number of scanner positions, while taking into account the previously mentioned constraints which are the angle of incidence, and max spacing between points. One restriction must be taken into account which is that the distance between the scanner and the scanned surface must be the same for all scanner positions.
The optimization module indicates the optimum scanning positions based on two objective functions. It utilizes three different optimization algorithms which are genetic algorithm, Jaya algorithm and particle swarm optimization. A fair comparison is then obtained between the three algorithms using some performance metrics such as coverage, and hypervolume indicators. Finally, weighted sum multi-criteria decision-making technique is implemented to determine the best solution and provide a ranking for all solutions. This research can be extended in the future to scan facades with non-plane surfaces.

\section{Acknowledgements}

This research was funded by Egypt-UK Newton-Musharafa Fund: Institutional Links; STDF (the Science \& Technology Development Fund), Egypt, Grant No. 26150.

\section{Funding}

This research was funded by Egypt-UK Newton-Musharafa Fund: Institutional Links; STDF (the Science \& Technology Development Fund), Egypt, Grant No. 26150.

\section{Disclosure statement}

Authors declare that they do not they have any competing financial, professional, or personal interests from other parties.

\section{References}

Ahn, J., \& Wohn, K. (2015). Interactive scan planning for heritage recording. Multimedia Tools and Applications, 75(7), 3655-3675. https://doi.org/10.1007/s11042-015-2473-0

Alshawabkeh, Y., \& Haala, N. (2004). Laser scanning and photogrammetry: A hybrid approach for heritage documentation. Paper presented at Third International Conference on Science \& Technology in Archaeology \& Conservation, The Hashimite University, Jordan.

Antón, D., Medjdoub, B., Shrahily, R., \& Moyano, J. (2018). Accuracy evaluation of the semi-automatic $3 \mathrm{D}$ modeling for historical building information models. International Journal of Architectural Heritage, 12(5), 790-805.

https://doi.org/10.1080/15583058.2017.1415391 
Baik, A. (2017). From point cloud to Jeddah heritage BIM Nasif historical house - Case study. Digital Applications in Archaeology and Cultural Heritage, 4, 1-18. https://doi.org/10.1016/j.daach.2017.02.001

Balzani, M., Santopuoli, N., Grieco, A., \& Zaltron, N. (2004, October). Laser scanner 3D survey in archaeological field: The Forum of Pompeii. In International Conference on Remote Sensing Archaeology (pp. 169-175). Beijing, China.

Barber, D., Mills, J., \& Bryan, P. (2003). Towards a standard specification for terrestrial laser scanning of cultural heritage. CIPA International Archives for Documentation of Cultural Heritage, 19, 619-624.

Basantes, J., Godoy, L., Carvajal, T., Castro, R., Toulkeridis, T., Fuertes, W., Aguilar, W., Tierra, A., Padilla, O., Mato, F., \& Ordoez, E. (2017, October). Capture and processing of geospatial data with laser scanner system for 3D modeling and virtual reality of Amazonian caves. In 2017 IEEE Second Ecuador Technical Chapters Meeting (ETCM). Salinas, Ecuador. https://doi.org/10.1109/ETCM.2017.8247455

Beume, N., Fonseca, C.M., López-Ibáñez, M., Paquete, L., \& Vahrenhold, J. (2009). On the complexity of computing the hypervolume indicator. IEEE Transactions on Evolutionary Computation, 13(5), 1075-1082.

https://doi.org/10.1109/TEVC.2009.2015575

Blaer, P. S., \& Allen, P. K. (2009). View planning and automated data acquisition for three-dimensional modeling of complex sites. Journal of Field Robotics, 26(11-12), 865-891.

https://doi.org/10.1002/rob.20318

Cabo, C., Ordóñez, C., \& Argüelles-Fraga, R. (2017). An algorithm for optimizing terrestrial laser scanning in tunnels. Automation in Construction, 83, 163-168. https://doi.org/10.1016/j.autcon.2017.08.028

Cabrelles, M., Galcerá, S., Navarro, S., Lerma, J. L., Akasheh, T., \& Haddad, N. (2009, October). Integration of 3D laser scanning, photogrammetry and thermography to record architectural monuments. In Proceedings of the 22nd International CIPA Symposium. Kyoto, Japan.

Calin, M., Damian, G., Popescu, T., Manea, R., Erghelegiu, B., \& Salagean, T. (2015). 3D modeling for digital preservation of Romanian heritage monuments. Agriculture and Agricultural Science Procedia, 6, 421-428.

https://doi.org/10.1016/j.aaspro.2015.08.111

Deb, K., Pratap, A., Agarwal, S., \& Meyarivan, T. A. M. T. (2002). A fast and elitist multiobjective genetic algorithm: NSGAII. IEEE Transactions on Evolutionary Computation, 6(2), 182-197. https://doi.org/10.1109/4235.996017

El-Hakim, S. F., Beraldin, J. A., Picard, M., \& Godin, G. (2004). Detailed 3D reconstruction of large-scale heritage sites with integrated techniques. IEEE Computer Graphics and Applications, 24(3), 21-29. https://doi.org/10.1109/MCG.2004.1318815

Fernández-Palacios, B. J., Morabito, D., \& Remondino, F. (2017). Access to complex reality-based 3D models using virtual reality solutions. Journal of Cultural Heritage, 23, 40-48. https://doi.org/10.1016/j.culher.2016.09.003

Forte, M., Pescarin, S., Pietroni, E., \& Dell'Unto, N. (2004). An integrated approach to archaeology: from the fieldwork to virtual reality systems. In F. Nicolucci \& S. Hermon (Eds.), Beyond the artifact. Digital interpretation of the past. Proceedings of CAA2004 (pp. 325-334). Archaeolingua, Budapest.

Giussani, A., \& Scaioni, M. (2004). Application of TLS to support landslides study: survey planning, operational issues and data processing. International Archives of Photogrammetry, Remote Sensing and Spatial Information Sciences, 36(8/W2), 318-323.
Grussenmeyer, P., Landes, T., Voegtle, T., \& Ringle, K. (2008). Comparison methods of terrestrial laser scanning, photogrammetry and tacheometry data for recording of cultural heritage buildings. International Archives of Photogrammetry, Remote Sensing and Spatial Information Sciences, 37(B5), 213-218.

Guidi, G., Russo, M., Ercoli, S., Remondino, F., Rizzi, A., \& Menna, F. (2009). A multi-resolution methodology for the $3 \mathrm{D}$ modeling of large and complex archeological areas. International Journal of Architectural Computing, 7(1), 39-55. https://doi.org/10.1260/147807709788549439

Ikeuchi, K., Oishi, T., Takamatsu, J., Sagawa, R., Nakazawa, A., Kurazume, R., Nishino, K., Kamakura, M., \& Okamoto, Y. (2007). The great buddha project: Digitally archiving, restoring, and analyzing cultural heritage objects. International Journal of Computer Vision, 75(1), 189-208.

https://doi.org/10.1007/s11263-007-0039-y

Kersten, T. P., Mechelke, K., Lindstaedt, M., \& Sternberg, H. (2009). Methods for geometric accuracy investigations of terrestrial laser scanning systems. Photogrammetrie-Fernerkundung-Geoinformation, 4, 301-315.

https://doi.org/10.1127/1432-8364/2009/0023

Kersten, T. P., Tschirschwitz, F., \& Deggim, S. (2017). Development of a virtual museum including a $4 \mathrm{D}$ presentation of building history in virtual reality. The International Archives of Photogrammetry, Remote Sensing and Spatial Information Sciences, 42(2/W3), 361-367.

https://doi.org/10.5194/isprs-archives-XLII-2-W3-361-2017

Kersten, T. P., Tschirschwitz, F., Deggim, S., \& Lindstaedt, M. (2018a). Virtual reality for cultural heritage monuments from $3 \mathrm{D}$ data recording to immersive visualisation. In $\mathrm{Eu}$ ro-Mediterranean Conference (pp. 74-83). Springer, Cham. https://doi.org/10.1007/978-3-030-01765-1_9

Kersten, T. P., Tschirschwitz, F., Lindstaedt, M., \& Deggim, S. (2018b). The historic wooden model of Solomon's Temple: 3D recording, modelling and immersive virtual reality visualisation. Journal of Cultural Heritage Management and Sustainable Development, 8(4), 448-464.

https://doi.org/10.1108/JCHMSD-09-2017-0067

Kriegel, S., Rink, C., Bodenmüller, T., \& Suppa, M. (2015). Efficient next-best-scan planning for autonomous $3 \mathrm{D}$ surface reconstruction of unknown objects. Journal of Real-Time Image Processing, 10(4), 611-631.

https://doi.org/10.1007/s11554-013-0386-6

Lee, I. S., Lee, J. O., Park, H. J., \& Bae, K. H. (2010). Investigations into the influence of object characteristics on the quality of terrestrial laser scanner data. KSCE Journal of Civil Engineering, 14(6), 905-913.

https://doi.org/10.1007/s12205-010-0986-7

MATLAB. (2018). MathWorks - Makers of MATLAB and Simulink 2019. https://www.mathworks.com/

Matthias, K., Severin, T., \& Salzwedel, H. (2013). Variable mutation rate at genetic algorithms: introduction of chromosome fitness in connection with multi-chromosome representation. International Journal of Computer Applications, 72(17), 31-38.

Rao, R. V., Rai, D. P., \& Balic, J. (2017). A multi-objective algorithm for optimization of modern machining processes. Engineering Applications of Artificial Intelligence, 61, 103-125. https://doi.org/10.1016/j.engappai.2017.03.001

Remondino, F. (2011). Heritage recording and 3D modeling with photogrammetry and 3D scanning. Remote Sensing, 3(6), 1104-1138. https://doi.org/10.3390/rs3061104 
Sánchez-Aparicio, L. J., Del Pozo, S., Ramos, L. F., Arce, A., \& Fernandes, F. M. (2018). Heritage site preservation with combined radiometric and geometric analysis of TLS data. Automation in Construction, 85, 24-39.

https://doi.org/10.1016/j.autcon.2017.09.023

Shanoer, M. M., \& Abed, F. M. (2018). Evaluate 3D laser point clouds registration for cultural heritage documentation. The Egyptian Journal of Remote Sensing and Space Science, 21(3), 295-304. https://doi.org/10.1016/j.ejrs.2017.11.007

Shao, J., Zhang, W., Mellado, N., Grussenmeyer, P., Li, R., Chen, Y., Wan, P., Zhang, X., \& Cai, S. (2019). Automated markerless registration of point clouds from TLS and structured light scanner for heritage documentation. Journal of Cultural Heritage, 35, 16-24. https://doi.org/10.1016/j.culher.2018.07.013

Song, M., Shen, Z. \& Tang, P. (2014). Data quality-oriented 3D laser scan planning. In Construction Research Congress 2014: Construction in a Global Network (pp. 984-993). Atlanta, Georgia, USA. https://doi.org/10.1061/9780784413517.101

Soudarissanane, S., \& Lindenbergh, R. (2011). Optimizing terrestrial laser scanning measurement set-up. International Archives of the Photogrammetry, Remote Sensing and Spatial Information Sciences, 38(5/W12), 127-132.

https://doi.org/10.5194/isprsarchives-XXXVIII-5-W12-127-2011

Soudarissanane, S., Lindenbergh, R., Menenti, M., \& Teunissen, P. J. G. (2009, September). Incidence angle influence on the quality of terrestrial laser scanning points. In Proceedings ISPRS Workshop Laserscanning. Paris, France. ISPRS.

Tan, B., Cai, Y., Zhang, Y., Wu, X., Chen, Y., \& Yang, B. (2016). Virtual reality continuum for heritage at Haw Par Villa in Singapore. In Proceedings of the Symposium on VR Culture and Heritage (pp. 71-74). Zhuhai, China. https://doi.org/10.1145/3014027.3014030
Tang, P., \& Alaswad, F. S. (2012). Sensor modeling of laser scanners for automated scan planning on construction jobsites. In Construction Research Congress 2012: Construction Challenges in a Flat World (pp. 1021-1031). West Lafayette, Indiana, United States. https://doi.org/10.1061/9780784412329.103

Tschirschwitz, F., Büyüksalih, G., Kersten, T. P., Kan, T., Enc, G., \& Baskaraca, P. (2019). Virtualising an Ottoman Fortress -Laser scanning and 3D modelling for the development of an interactive, immersive virtual reality application. International Archives of the Photogrammetry, Remote Sensing and Spatial Information Sciences, 42(2/W9), 723-729.

https://doi.org/10.5194/isprs-archives-XLII-2-W9-723-2019

Windarto, A. P., \& Muhammad, A. (2017). Comparison of weighted sum model and multi attribute decision making weighted product methods in selecting the best elementary school in Indonesia. International Journal of Software Engineering and its Applications, 11(4), 69-90. https://doi.org/10.14257/ijseia.2017.11.4.06

Yastikli, N. (2007). Documentation of cultural heritage using digital photogrammetry and laser scanning. Journal of Cultural Heritage, 8(4), 423-427. https://doi.org/10.1016/j.culher.2007.06.003

Zhang, C., Kalasapudi, V. S., \& Tang, P. (2016). Rapid data quality oriented laser scan planning for dynamic construction environments. Advanced Engineering Informatics, 30(2), 218-232. https://doi.org/10.1016/j.aei.2016.03.004

Zhang, H., \& Li, H. (2010). Multi-objective particle swarm optimization for construction time-cost tradeoff problems. Construction Management and Economics, 28(1), 75-88. https://doi.org/10.1080/01446190903406170 